\title{
Prognostic value of lung ultrasonography and bioimpedance spectroscopy in patients with heart failure and reduced ejection fraction
}

\author{
Dimitrie Siriopol ${ }^{1,2}$, Mihaela Siriopol $^{1}$, Mihaela Mihaila ${ }^{2}$, Florentina Rusu², Radu Sascau ${ }^{3}$, \\ Irina Costache ${ }^{3}$, Vlad Vasiliu², Andreea Bucur², Andreea Neamtu², Raluca Popa ${ }^{1,2}$, Petru Cianga ${ }^{4}$, \\ Mehmet Kanbay ${ }^{5}$, Adrian Covic ${ }^{1,2}$
}

\author{
'Department of Nephrology, University of Medicine and Pharmacy "Grigore T. Popa”, \\ Iasi, Romania \\ 2“Dr. C.I. Parhon” University Hospital, Iasi, Romania \\ ${ }^{3}$ Department of Cardiology, University of Medicine and Pharmacy "Grigore T. Popa”, \\ Iasi, Romania \\ 4Department of Immunology, University of Medicine and Pharmacy “Grigore T. Popa”, \\ lasi, Romania \\ ${ }^{5}$ Division of Nephrology, Department of Medicine, Koc University School of Medicine, \\ Istanbul, Turkey
}

Submitted: 21 October 2019

Accepted: 25 January 2020

Arch Med Sci

DOI: https://doi.org/10.5114/aoms.2020.95727

Copyright (c) 2020 Termedia \& Banach

\begin{abstract}
Introduction: Chronic heart failure (HF) represents a major global public health problem, and despite significant advances in diagnosis and management over the past two decades, HF patients still have a poor prognosis. The aim of the study was to evaluate the relationship between lung congestion, as assessed by lung ultrasonography (LUS), bioimpedance spectroscopy, body fluid compartments, and echocardiographic parameters, and to determine the effect of these associations on all-cause mortality in HF patients.

Material and methods: Eligible patients with a left ventricular ejection fraction (LVEF) below $45 \%$ were identified via daily echocardiography assessments. Lung ultrasonography was performed with patients in the supine position, for a total of 28 sites per complete examination. The extracellular water (ECW) was determined using a BIS device.

Results: Our study included 122 patients (67.2\% males) with a mean age of 67.2 years. In the multivariable linear regression analysis, including all the univariable predictors of lung congestion, only New York Heart Association (NYHA) class, ECW, estimated glomerular filtration rate (eGFR), and LVEF levels maintained an independent association with the number of B-lines. During the follow-up, 33 patients died. In multivariable Cox analysis, a B-line number of at least 15 remained significantly associated with all-cause mortality, independently of age, sex, diabetes, LVEF, estimated glomerular filtration rate, $\mathrm{C}$-reactive protein, $\mathrm{N}$-terminal pro-brain natriuretic peptide, or ECW values (adjusted HR $=3.84,95 \% \mathrm{Cl}: 1.12-13.09$ ).

Conclusions: We show for the first time in HF patients that pulmonary congestion, as assessed by LUS, is associated with the severity of NYHA class, LVEF, eGFR, and ECW, and it identifies those at increased risk of death.
\end{abstract}

Key words: lung ultrasonography, B-line, bioimpedance spectroscopy, extracellular water, heart failure, mortality.
Corresponding author: Dimitrie Siriopol

Carol I Bld

700503, Iasi, Romania

E-mail:

dimitrie.siriopol@yahoo.com 


\section{Introduction}

Chronic heart failure (HF) represents a major global public health problem. In the United States, approximately 6.2 million adult Americans have chronic HF, with estimations that show an increase to more than 8 million people in 2030 [1]. Similar findings were also observed in European populations [2, 3]. Despite important advances in diagnosis and management over the past two decades [4], chronic HF patients still have a poor prognosis $[1,5-8]$ with a mortality around $50 \%$ at five years [9].

Lung congestion is one of the most common signs of HF, being also the leading cause for hospitalisation in adult patients over 65 years from the United States [10]. Traditionally, lung congestion has been evaluated by clinical examination or thoracic radiography, but in recent years lung ultrasonography (LUS) has emerged as a superior diagnostic test in HF patients [11]. Furthermore, LUS, through the assessment of the B-lines, is also a promising tool for monitoring changes in lung congestion [12] and for prognostic stadialisation $[12,13]$ in these patients. The B-lines are discrete laser-like vertical hyperechoic reverberation artefacts that arise from the pleural line, extend to the bottom of the screen without fading, and move in tandem with lung sliding [14], originating most often from volumetric variations in the relationship between the aerated and tissue/ fluid-filled parts of the lung [15].

Bioimpedance spectroscopy (BIS) represents an objective tool that is used to assess and monitor fluid status. It is validated against "gold-standard" methods [16, 17], and its use in dialysis patients has been associated with improved survival [18], better blood pressure control [19], and reduced arterial stiffness [18].

The aim of the study was to evaluate the relationship between lung congestion (as assessed by LUS), BIS-derived and echocardiographic parameters, and to determine the effect of these associations on all-cause mortality in HF patients.

\section{Material and methods}

\section{Patients and study design}

This was a prospective observational study of outpatient adults referred for clinically indicated transthoracic echocardiograms at an academic hospital between 2016 and 2018. Eligible patients with a left ventricular ejection fraction (LVEF) below $45 \%$ were identified via the daily echocardiography assessments. From a total of 264 eligible patients, we excluded $127 \mathrm{pa}$ tients because of limb amputation $(n=3)$, metallic joint prostheses $(n=10)$, cardiac pacemakers or stents $(n=78)$, decompensated cirrhosis $(n=5)$, prior diagnosis of pulmonary fibrosis $(n=4)$, pneumectomy $(n=1)$, massive pleural effusion $(n=6)$, end-stage renal disease $(n=3)$, active systemic infections $(n=4)$, and terminal illnesses $(n=13)$. There were an additional 15 patients who refused to participate and were not included in the study. Details of the final population $(n=122)$ are shown in Tables I, II and Supplementary Table I.

The investigation conforms with the principles outlined in the Declaration of Helsinki [20]. The study was approved by the Research Ethics Committee of the "Grigore T. Popa" University of Medicine and Pharmacy lasi, and all included patients signed an informed consent form. The trial was registered at ClinicalTrials.gov, NCT02764073.

\section{Demographic and clinical parameters}

The following demographic parameters were recorded at baseline: age, gender, weight, height, comorbidities (diabetes, coronary artery disease, hypertension, atrial fibrillation, chronic kidney disease [CKD]), and smoking status. Arterial blood pressure was determined in the morning in all patients by a physician by three consecutive measurements, after a 15-minute resting period, with the mean values calculated for systolic (SBP) and diastolic blood pressure (DBP). We used an automatic BP measuring device certified by the Association for the Advancement of Medical Instrumentation, European Society of Hypertension, and British Society of Hypertension - model OMRON M6, with an adjustable cuff for arm circumferences from 24 to $42 \mathrm{~cm}$, respecting the current guideline recommendations [21]. Hypertension was defined as a SBP of at least $140 \mathrm{~mm} \mathrm{Hg}$ and/ or diastolic BP of at least $90 \mathrm{~mm} \mathrm{Hg}$ or previously diagnosed hypertension under treatment during the previous two weeks, regardless of BP values. We considered that a patient had coronary artery disease if in the medical records there was a diagnosis of coronary artery/heart disease, angina or angina pectoris, or myocardial infarction. Chronic kidney disease was defined according to the Kidney Disease Improving Global Outcomes guidelines [22].

We also evaluated diuresis, the presence of peripheral oedema (slight pitting of at least $2 \mathrm{~mm}$ depth with no visible distortion) [23], the New York Heart Association (NYHA) functional class, and the medication.

\section{Biochemical analysis}

All blood samples were obtained from patients in the morning, after 12 hours of fasting, for measurement of serum creatinine, haemoglo- 
Table I. Baseline demographic, clinical, and biological characteristics of the study population

\begin{tabular}{|c|c|c|c|c|}
\hline Parameter & Total $(N=122)$ & Group $1(n=57)$ & Group $2(n=65)$ & $P$ value \\
\hline Age [years] & $67.2 \pm 11.0$ & $65.1 \pm 9.9$ & $68.9 \pm 12.1$ & 0.06 \\
\hline BMI $\left[\mathrm{kg} / \mathrm{m}^{2}\right]$ & $29.2 \pm 5.3$ & $29.1 \pm 4.7$ & $29.3 \pm 5.8$ & 0.88 \\
\hline Male, $n[\%]$ & $82(67.2)$ & $42(73.7)$ & $40(61.5)$ & 0.15 \\
\hline $\mathrm{SBP}[\mathrm{mm} \mathrm{Hg}]$ & $124.6 \pm 18.7$ & $125.1 \pm 17.2$ & $124.1 \pm 20.1$ & 0.78 \\
\hline $\mathrm{DBP}[\mathrm{mm} \mathrm{Hg}]$ & $75.0 \pm 11.3$ & $76.3 \pm 9.5$ & $73.8 \pm 12.7$ & 0.22 \\
\hline Smoking, $n[\%]$ & $34(27.9)$ & $15(26.3)$ & $19(29.2)$ & 0.72 \\
\hline Diuresis [l/day] & $1.4 \pm 0.5$ & $1.3 \pm 0.5$ & $1.4 \pm 0.5$ & 0.24 \\
\hline Oedema, $n[\%]$ & $66(54.1)$ & $17(29.8)$ & $49(75.4)$ & $<0.001$ \\
\hline III/IV NYHA class, $n$ [\%] & $66(54.1)$ & $13(22.8)$ & $53(81.5)$ & $<0.001$ \\
\hline B-lines & $16.0(4.0-35.0)$ & $3.0(1.0-9.0)$ & $34.0(23.0-51.0)$ & $<0.001$ \\
\hline \multicolumn{5}{|l|}{ Comorbidities } \\
\hline Diabetes, $n$ [\%] & $44(36.1)$ & $17(29.8)$ & $27(41.5)$ & 0.18 \\
\hline Coronary artery disease, $n[\%]$ & $67(54.9)$ & $31(54.4)$ & $36(55.4)$ & 0.91 \\
\hline Hypertension, $n$ [\%] & $77(63.1)$ & $40(70.2)$ & $37(56.9)$ & 0.13 \\
\hline Atrial fibrillation, $n$ [\%] & $71(58.2)$ & $34(59.7)$ & $37(56.9)$ & 0.76 \\
\hline CKD, $n[\%]$ & $32(26.2)$ & $11(19.3)$ & $21(32.3)$ & 0.10 \\
\hline \multicolumn{5}{|l|}{ Biological parameters } \\
\hline Serum creatinine $[\mathrm{mg} / \mathrm{dl}]$ & $1.1(0.9-1.4)$ & $1.0(0.9-1.2)$ & $1.2(0.9-1.5)$ & 0.02 \\
\hline EGFR $\left[\mathrm{ml} / \mathrm{min} / 1.73 \mathrm{~m}^{2}\right]$ & $66.2 \pm 24.2$ & $73.3 \pm 20.4$ & $59.9 \pm 25.6$ & 0.002 \\
\hline Haemoglobin [g/dl] & $13.2 \pm 2.1$ & $13.9 \pm 2.1$ & $12.6 \pm 1.9$ & $<0.001$ \\
\hline Serum glucose [mg/dl] & $106.0(97.0-131.0)$ & $104.0(95.0-128.0)$ & $107.0(99.0-134.0)$ & 0.33 \\
\hline Total cholesterol [mg/dl] & $153.3 \pm 44.1$ & $161.4 \pm 46.9$ & $146.2 \pm 40.4$ & 0.06 \\
\hline LDL cholesterol [mg/dl] & $87.5(70.0-115.0)$ & $97.0(76.0-125.0)$ & $85.0(69.0-101.0)$ & 0.12 \\
\hline HDL cholesterol [mg/dl] & $37.0(29.0-45.0)$ & $39.0(32.0-46.0)$ & $35.0(28.0-45.0)$ & 0.12 \\
\hline Serum triglycerides [mg/dl] & $124.0(96.0-148.0)$ & $122.0(96.0-148.0)$ & $125(97.0-147.0)$ & 0.54 \\
\hline CRP $[\mathrm{mg} / \mathrm{l}]$ & $27.0(11.2-57.8)$ & $18.2(6.5-49.5)$ & $35.0(18.2-108.0)$ & 0.01 \\
\hline Uric acid [mg/dl] & $7.4(6.2-8.8)$ & $7.0(6.2-7.9)$ & $7.8(6.2-9.2)$ & 0.03 \\
\hline Serum sodium [mmol/l] & $137.4 \pm 4.9$ & $138.9 \pm 3.2$ & $136.2 \pm 4.9$ & 0.001 \\
\hline NT-proBNP [pg/ml] & $\begin{array}{c}895.0 \\
(300.0-1600.0)\end{array}$ & $\begin{array}{c}600.0 \\
(200.0-1300.0)\end{array}$ & $\begin{array}{c}1080.0 \\
(500.0-1950.0)\end{array}$ & 0.002 \\
\hline
\end{tabular}

$B M I$ - body mass index, CKD - chronic kidney disease, CRP-C-reactive protein, DBP-diastolic blood pressure, eGFR-estimated glomerular filtration rate, $H D L$ - high-density lipoprotein, $L D L$ - low-density lipoprotein, NT-proBNP - N-terminal pro-brain natriuretic peptide, SBPsystolic blood pressure. CKD was defined according to the Kidney Disease Improving Global Outcomes guidelines [22].

bin, glucose, total cholesterol, triglycerides (TG), high-density lipoprotein (HDL), low-density lipoprotein (LDL) cholesterol, C-reactive protein (CRP), uric acid, and sodium levels. The estimated glomerular filtration rate (eGFR) was calculated using the Chronic Kidney Disease Epidemiology Collaboration (CKD-EPI) equation [24]. All laboratory tests were performed by standard procedures with cer- tified methods on the day of the lung ultrasonography and BIS assessments.

We also stored serum at $-80^{\circ} \mathrm{C}$ for subsequent analyses. $N$-Terminal Pro-Brain Natriuretic Peptide (NT-proBNP) in these samples was analysed using the Elabscience ${ }^{\oplus}$ ELISA Kit, an electro-chemiluminescence 'sandwich' immunoassay based on antibodies against Human NT-proBNP. 
Table II. Echocardiographic and bioimpedance characteristics of the study population

\begin{tabular}{|c|c|c|c|c|}
\hline Parameter & Total $(N=122)$ & Group $1(n=57)$ & Group $2(n=65)$ & $P$ value \\
\hline \multicolumn{5}{|l|}{ Echocardiography } \\
\hline LV EDVi $\left[\mathrm{ml} / \mathrm{m}^{2}\right]$ & $85.4(71.4-107.3)$ & $81.3(68.8-99.3)$ & 88.8 (76.4-110.9) & 0.04 \\
\hline LV ESVi $\left[\mathrm{ml} / \mathrm{m}^{2}\right]$ & $46.1(30.2-73.3)$ & $37.9(26.9-58.0)$ & $58.2(35.1-78.2)$ & 0.004 \\
\hline Septal wall thickness $[\mathrm{cm}]$ & $1.2(1.1-1.4)$ & $1.3(1.2-1.4)$ & $1.2(1.1-1.3)$ & 0.18 \\
\hline LV mass index $\left[\mathrm{g} / \mathrm{m}^{2}\right]$ & $154.4(131.6-187.3)$ & $148.3(131.4-173.4)$ & $164.9(132.3-192.7)$ & 0.17 \\
\hline LA volume index $\left[\mathrm{ml} / \mathrm{m}^{2}\right]$ & $43.6(33.6-68.5)$ & $39.4(32.8-58.5)$ & $54.1(34.7-74.0)$ & 0.03 \\
\hline LVEF (\%) & $32.8 \pm 9.1$ & $36.8 \pm 7.6$ & $29.3 \pm 8.9$ & $<0.001$ \\
\hline \multicolumn{5}{|l|}{ Bioimpedance } \\
\hline TBW [I] & $36.5 \pm 5.8$ & $36.4 \pm 5.9$ & $36.6 \pm 5.8$ & 0.85 \\
\hline ECW [l] & $18.3 \pm 2.9$ & $17.4 \pm 3.0$ & $19.1 \pm 2.7$ & 0.001 \\
\hline ICW [I] & $18.0 \pm 3.4$ & $18.7 \pm 3.6$ & $17.4 \pm 3.3$ & 0.04 \\
\hline
\end{tabular}

AFO - absolute fluid overload, ECW - extracellular water, ICW - intracellular water, LA - left atrial, LVEF - left ventricular ejection fraction, $L V E D D$ - LV end diastolic diameter, LV EDVi - left ventricular end diastolic volume index, LV ESD - LV end systolic diameter, LV ESVi - left ventricular end systolic volume index, RFO - relative fluid overload, TBW - total body water.

Table III. Univariable and multivariable associates of B-lines (log transformed) in the study population

\begin{tabular}{|c|c|c|}
\hline Parameter & Univariate $\boldsymbol{\rho}$ & $P$ \\
\hline Edema (0 - No, 1 - Yes) & 0.43 & $<0.001$ \\
\hline NYHA class (0 - II, 1 - III/IV) & 0.56 & $<0.001$ \\
\hline Diuretic use (0 - No, 1 - Yes) & 0.19 & 0.03 \\
\hline Log LV ESVi $\left[\mathrm{ml} / \mathrm{m}^{2}\right]$ & 0.19 & 0.03 \\
\hline Log LA volume index $\left[\mathrm{ml} / \mathrm{m}^{2}\right]$ & 0.23 & 0.01 \\
\hline Log LVEF (\%) & -0.33 & $<0.001$ \\
\hline ECW [I] & 0.29 & $<0.001$ \\
\hline ICW [I] & -0.17 & 0.04 \\
\hline $\mathrm{EGFR}\left[\mathrm{ml} / \mathrm{min} / 1.73 \mathrm{~m}^{2}\right]$ & -0.33 & $<0.001$ \\
\hline Log CRP [mg/l] & 0.23 & 0.01 \\
\hline Serum sodium [mmol/l] & -0.32 & $<0.001$ \\
\hline \multirow[t]{2}{*}{ Log NT-proBNP [pg/ml] } & 0.35 & $<0.001$ \\
\hline & Multivariate $\boldsymbol{\beta}$ & $P$ \\
\hline NYHA class (0 - II, 1 - III/IV) & 1.11 & $<0.001$ \\
\hline Log LVEF (\%) & -0.61 & 0.04 \\
\hline ECW [l] & 0.08 & 0.01 \\
\hline $\mathrm{EGFR}\left[\mathrm{ml} / \mathrm{min} / 1.73 \mathrm{~m}^{2}\right]$ & -0.01 & 0.001 \\
\hline
\end{tabular}

$C R P$ - C-reactive protein, ECW - extracellular water, eGFR estimated glomerular filtration rate, ICW - intracellular water, LA - left atrial, LVEF - left ventricular ejection fraction, LV ESVi - left ventricular end systolic volume index, NT-proBNP - N-terminal probrain natriuretic peptide, TBW - total body water.

\section{Echocardiographic assessment}

Echocardiographic measurements were performed before (the same day or maximum one day before) LUS and BIS evaluations by two trained echocardiographers according to the recommendations of the American Society of Echocardiography [25].

\section{B-line assessment}

Lung ultrasonography was performed with patients in the supine position, for a total of 28 sites per complete examination, as previously described [26]. At every scanning site, B-lines could be counted from 0 (no B-lines) to 10 (complete white screen) (Supplementary Figure 1). The sum of the B-lines gave a score representing the extent of extravascular lung water. Two trained physicians blinded to the echocardiography assessment performed all measurements.

\section{Bioimpedance analysis}

The hydration state and the body composition were assessed immediately after the LUS evaluation using a portable whole-body BIS device (BCM - Fresenius Medical Care D GmbH [27]). By attaching electrodes to the patient's forearm and ipsilateral ankle, this device measures the impedance spectroscopy at 50 frequencies. The extracellular water (ECW), intracellular water (ICW), and TBW were determined as previously described [28].

\section{Outcome}

The main outcome was all-cause mortality. Death was confirmed through patient follow-up 
phone calls, the hospital's electronic medical records, or the social security death index.

\section{Statistical analysis}

Variables were expressed as median with interquartile range $(\mathrm{IQR})$, mean \pm standard deviation (SD), or as percentage of frequency, as appropriate. For the categorical variables, the between-group comparisons were performed using the $\chi^{2}$ test, and for the continuous variables using the Mann-Whitney $U$ test or the independent $T$-test, as appropriate. The Shapiro-Wilk test was used for assessing the normality of the distribution and logarithmic conversion was performed for non-normally distributed variables, including B-lines number, glucose, HDL and LDL-cholesterol, triglycerides, CRP, and uric acid levels.

Pearson's correlation coefficient was used to determine correlations between variables. Backward stepwise multivariable regression analysis including all univariate associates $(p<0.05)$ was used to assess the predictors for B-lines.

Time-to-event analysis of all-cause mortality was performed using Kaplan-Meier cumulative survival plots and Cox proportional hazards model, including adjustment for potential confounding factors. In model 1, we adjusted for clinical risk factors: age, sex, and diabetes. In model 2, we adjusted for biochemical and echocardiographic (LVEF) risk factors: CRP, eGFR, NT-proBNP, and LVEF. In model 3, we adjusted for all the variables used in previous two models. We also made an additional model (model 4) in which we adjusted also for ECW (for the B-line analysis) or B-lines (for the ECW analysis).

To determine the optimal cut-off point for the number of B-lines and ECW as predictors of all-cause mortality, the relationship between the number of B-lines and ECW and the outcome was analysed using the Martingale residuals in Cox's proportional hazard regression analysis [29]. We found that the categorisation of the number of B-lines and ECW into two categories (using 15 B-lines and 18.3 I as cut-offs for the number of B-line and ECW, respectively) was the best approach for modelling these relationships. Analyses were performed with B-lines and ECW as a categorical (based on the cut-offs mentioned above) and also as a continuous variable. Data are presented in the form of Hazard ratios and $95 \%$ confidence intervals. We tested the proportional hazards assumptions using the Schoenfeld residuals, and when needed we performed bootstrapping validation in order to avoid the problem of over fitting due to the low number of death events.

All analyses were performed using Stata SE software, version 13 (StatCorp, College Station, TX, USA) and the R (version 3.6.1) package for statisti- cal analysis (Foundation for Statistical Computing, Vienna, Austria). A $p$ value $<0.05$ was considered to be statistically significant.

\section{Results}

\section{Characteristics of the study population}

Our study included 122 patients (67.2\% males) with a mean age of 67.2 years. The most frequent cause of HF was dilated cardiomyopathy - 47 (43.1\%) patients. However, the exact cause of HF was unknown in 25 (20.5\%) patients. More than half had at least NYHA class III; the median number of B-lines was 16 . Other characteristics of the population are presented in Tables I and II and Supplementary Table I.

As a first step we divided the study patients according to the number of B-lines (Group 1 B-lines below 15 and Group 2 B-lines with at least 15 B-lines). As expected, patients in Group 1 $B$-lines had a lower prevalence of oedema and less severe NYHA class; they also showed better renal function (as assessed by eGFR) and lower CRP, NT-proBNP, and uric acid levels than patients from Group 2 B-lines (Table I). Furthermore, these patients had lower left atrial and left ventricular volumes and a better LVEF than patients from Group 2 B-lines (see Table II). Bioimpedance spectroscopy fluid status assessment showed that although there was no significant difference between these two groups in TBW, patients from Group 1 B-lines had lower ECW and higher ICW volumes (Table II).

\section{Correlations of lung congestion}

with demographic, clinical, biological, and bioimpedance parameters

In univariable analysis, we identified eGFR, CRP, serum sodium, NT-proBNP, and different clinical, echocardiographic, and BIS derived characteristics as significant correlates for the number of B-lines. In multivariable linear regression analysis, including all the univariable predictors of lung congestion, only NYHA class, ECW, eGFR, and LVEF levels maintained an independent association ( $R^{2}$ of the model $=0.42$ ) with the number of B-lines (see Table III).

\section{All-cause mortality according to the number of B-lines and extracellular water volume}

During the follow-up (mean 12.5 months, median 11.2 months), 33 patients (27.1\%) died. There were seven deaths in the group of patients with the number of B-lines below 15, while in the other group there were 26 deaths (there were 12 deaths in the group of patients with below 18.3 I of ECW 
A

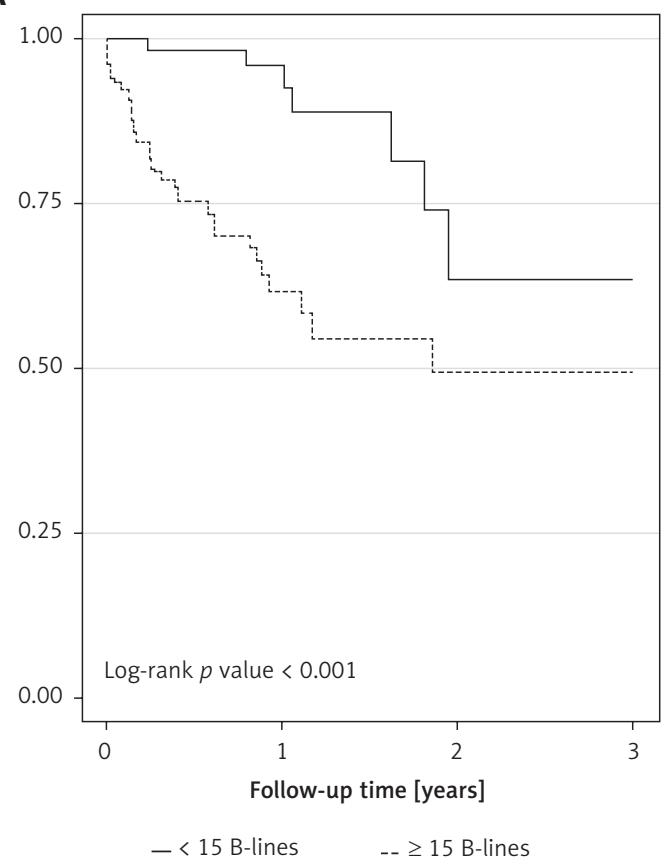

B

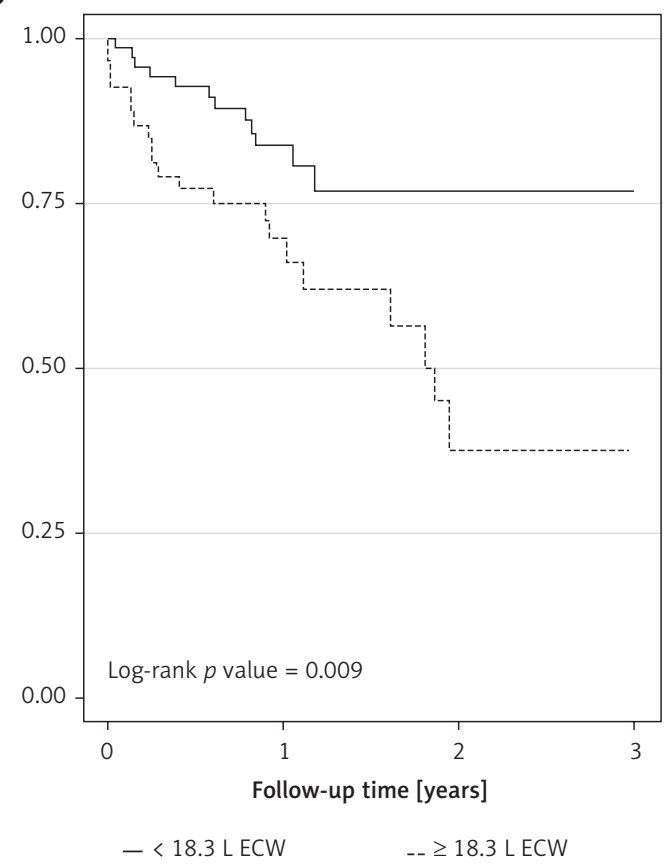

Figure 1. Kaplan-Meier curves for all-cause mortality according to the B-lines (A) and ECW (B) groups

and 21 deaths in the group with at least 18.3 of ECW). Kaplan-Meier curves show significantly higher all-cause mortality in HF patients with at least 15 B-lines (see Figure 1 A) or 18.3 I ECW volume (Figure $1 \mathrm{~B}$ ). In unadjusted Cox regression, patients from Group 2 B-lines had a HR of 3.96 (95\% Cl: 1.78-8.79), while those in Group 2 ECW had a HR for death of 2.47 (95\% Cl: 1.23-4.98) for the outcome of interest (see Table IV). In multivariable Cox analysis, a number of B-lines of at least 15 remained significantly associated with all-cause mortality, independently of different clinical, echocardiographic, biological, or BIS-derived fluid characteristics (Table IV). However, an 18.3 I of ECW volume remained significantly associated with the outcome after adjustments for clinical, echocardiographic, or biological risk factors (Table IV, Models 1 and 2) but lost this significance after adjustment for all the aforementioned risk factors (Table IV, Model 3) or for lung congestion (Table IV, Model 4).

Using the number of B-lines and ECW as continuous variables, we found in univariate Cox analysis that both variables were associated with an elevated risk of death $(\mathrm{HR}=2.04,95 \% \mathrm{Cl}: 1.36-3.08$ and $\mathrm{HR}=1.16,95 \% \mathrm{Cl}: 1.03-1.29$ for each increment of 1 SD in log number of B-lines and 11 in ECW volume, respectively). After adjustment, the number of B-lines remained significantly associated with the outcome in all the multivariable Cox models, while ECW lost the significance for this relationship after adjustment for biological characteristics and LVEF (Supplementary Table II).

\section{Discussion}

This study shows for the first time that, in HF patients with a reduced LVEF, lung congestion is dependent on eGFR, LVEF, and ECW volume, as assessed by BIS. We also confirm the increased risk of death associated with pulmonary congestion, but we show that this association is independent of cardiac and renal function or body fluid compartments.

Congestion is the main feature and reason for hospital admission of HF patients. However, the physiopathological transition from haemodynamic congestion (increased left ventricular filling pressures) to lung congestion (increased extravascular lung water) and finally to clinical congestion (signs and symptoms of congestion) is complex and still incompletely understood. Extravascular lung water, a relatively small component of body fluids compartments, is usually related to increased left ventricular filling pressures and total body fluids. Some studies support the idea that pulmonary congestion is related more to fluid redistribution than to total body fluid accumulation. In the IMPACT-HP trial the authors demonstrated that the degree of weight loss was not associated with clinical improvement [30]. Similarly, in the EVEREST study the weight reduction obtained with tolvaptan was not associated with improvements in global clinical status [31]. Our study confirms this hypothesis and adds new data into this puzzle. We show that although there is no difference in TBW between the patients with 
Table IV. Lung congestion and extracellular water as predictors of all-cause mortality (using the B-lines and extracellular water groups)

\begin{tabular}{|c|c|c|c|c|}
\hline & \multicolumn{2}{|c|}{ B-lines } & \multicolumn{2}{|c|}{ ECW } \\
\hline & $H R^{*}$ & $95 \% \mathrm{Cl}$ & $\mathrm{HR}^{*}$ & $95 \% \mathrm{Cl}$ \\
\hline Unadjusted & 3.96 & $1.78-8.79$ & 2.47 & $1.23-4.98$ \\
\hline \multicolumn{5}{|l|}{ Adjusted } \\
\hline Model 1 & 4.88 & $1.66-14.34$ & 2.19 & $1.07-4.48$ \\
\hline Model 2 & 3.58 & $1.55-8.29$ & 2.24 & $1.09-4.59$ \\
\hline Model 3 & 3.87 & $1.16-12.91$ & 1.99 & $0.94-4.18$ \\
\hline Model 4 & 3.84 & $1.12-13.09$ & 1.62 & $0.75-3.51$ \\
\hline
\end{tabular}

Cl-confidence interval, ECW - extracellular water, HR - hazard ratio. Model 1: age, sex, diabetes. Model 2: C-reactive protein, estimated glomerular filtration rate, left ventricular ejection fraction, N-terminal pro-brain natriuretic peptide. Model 3: variables in model 1 + variables in model 2. Model 4: Model 3 + ECW (for the B-lines groups) or Model 3 + B-lines (for the ECW groups). *Hazard ratio for Group 2 vs. Group 1

below and those with at least 15 B-lines, there are significantly higher ECW volumes in patients from the latter group. Furthermore, in the multivariable regression model, ECW is one of the variables that remained independently associated with lung congestion.

Another important piece in the puzzle is the relationship between eGFR, as a surrogate of renal function, and the degree of pulmonary congestion in HF patients. We show an inverse and independent association between eGFR and the number of B-lines. There is a known bidirectional lung-kidney crosstalk commonly present in health and specific pathological states [32]. As seen in acute kidney injury or CKD, there are elevations in the levels of different mediators that could alter pulmonary vascular permeability [32] and as such increase the risk of pulmonary congestion. However, also pulmonary congestion with impaired gas exchange could lead to decreased renal blood flow and alterations in eGFR through stimulation of adrenergic nerves and disturbances in nitric oxide metabolism [33]. Although in this relationship the primum movens is not known, our results clinically validate the increased risk of pulmonary congestion related to worse renal function in $\mathrm{HF}$ patients with reduced LVEF.

It is now well established that LUS, through the assessment of B-lines, is a practical diagnostical and prognostic tool in acute and chronic $\mathrm{HF}$ patients [12]. Findings from studies performed in chronic HF patients suggest that those with pulmonary congestion are at an increased risk of hospitalisation or death [13, 34-36]. With a fourfold increase in the risk for all-cause mortality, our findings are consistent with these studies; however, by comparison with the aforementioned studies, although the authors adjusted the results for demographic [34-36], clinical [13, 35], or cardiac characteristics $[13,34]$, our study is the only one that combines demographical clinical, cardiac, biological, and, most importantly, BIS-derived fluid status confounders into the survival analysis. The fact that the number of B-lines is associated with all-cause mortality even after all these adjustments reinforces the important role played by pulmonary congestion in predicting adverse outcomes in HF patients. Our cut-off of 15 B-lines, derived from statistical analysis, is the same as the clinical cut-off proposed and used in previous studies [12, 13].

Bioimpedance spectroscopy has shown promising results, especially in dialysis patients [18], in HF patients its use being limited to only two diagnostic studies performed in an acute setting with no follow-up outcomes [37, 38]. Although our study shows that an ECW higher than 18.3 I is associated with death in the initial multivariable Cox models, after different adjustments, including also pulmonary congestion, this association becomes non-significant. We obtained similar findings in a previous study performed in dialysis patients. In the final model, the number of B-lines, and not the BIS derived fluid status parameter, was associated with all-cause mortality [39].

This study supports previous findings regarding the impact of lung congestion on all-cause mortality in HF patients, but at the same time refines this association. We used a holistic approach in our analysis, trying to underline the complex relationship between the lung, heart, and kidney and its effect on survival in this type of patient.

\section{Limitations and strengths}

Our study has some limitations that must be addressed. We included a relatively small sample size of selected HF patients from a single centre with a relatively low number of mortality events, but we used different statistical approaches to overcome these shortcomings. Furthermore, we 
are not able to completely exclude the existence of residual confounding in our regression models. These factors could limit the generalisability of the results obtained in this study. However, our findings are particularly encouraging because they emphasise the complex pathways that link lung congestion with cardiac and renal function and body fluid compartments. Ideally, these results should be validated in other HF populations, including larger samples. In addition, we could not specify the cause of death, and the data about nonfatal events were not collected.

In conclusion, we show for the first time in HF patients that pulmonary congestion, as assessed by lung ultrasonography, is associated with the severity of NYHA class, LVEF, eGFR, and ECW, and it identifies those at increased risk of death. Further studies are required to determine whether such early warnings, such as pulmonary or systemic fluid congestion, may prevent the clinical progression of disease and HF hospitalisation.

\section{Acknowledgements}

This work was supported by a grant of the Ministery of Research and Innovation, CNCS-UEFISCDI, project number PN-III-P1-1.1-PD-2016-0287, within PNCDI III and by a grant of the "Grigore T. Popa" University of Medicine and Pharmacy, contract number 27505/2018.

\section{References}

1. Benjamin EJ, Muntner P, Alonso A, et al.; American Heart Association Council on Epidemiology and Prevention Statistics Committee and Stroke Statistics Subcommittee. Heart disease and stroke statistics - 2019 update: a report from the American Heart Association. Circulation 2019; 139: e56-e528.

2. Ohlmeier C, Mikolajczyk R, Frick J, Prutz F, Haverkamp W, Garbe E. Incidence, prevalence and 1-year all-cause mortality of heart failure in Germany: a study based on electronic healthcare data of more than six million persons. Clin Res Cardiol 2015; 104: 688-96.

3. Buja A, Solinas G, Visca M, et al. Prevalence of heart failure and adherence to process indicators: which socio-demographic determinants are involved? Int J Environ Res Public Health 2016; 13: 238.

4. Michalska-Kasiczak M, Bielecka-Dabrowa A, von Haehling S, Anker SD, Rysz J, Banach M. Biomarkers, myocardial fibrosis and co-morbidities in heart failure with preserved ejection fraction: an overview. Arch Med Sci 2018; 14: 890-909.

5. Arundel C, Sheriff H, Bearden DM, et al. Discharge home health services referral and 30-day all-cause readmission in older adults with heart failure. Arch Med Sci 2018; 14: 995-1002.

6. Beiert T, Straesser S, Malotki R, Stockigt F, Schrickel JW, Andrie RP. Increased mortality and ICD therapies in ischemic versus non-ischemic dilated cardiomyopathy patients with cardiac resynchronization having survived until first device replacement. Arch Med Sci 2019; 15: $845-56$.
7. Orimoloye OA, Kambhampati S, Hicks AJ 3rd, et al. Higher cardiorespiratory fitness predicts long-term survival in patients with heart failure and preserved ejection fraction: the Henry Ford Exercise Testing (FIT) Project. Arch Med Sci 2019; 15: 350-8.

8. Polikandrioti M, Panoutsopoulos G, Tsami A, et al. Assessment of quality of life and anxiety in heart failure outpatients. Arch Med Sci Atheroscler Dis 2019; 4: e38-e46.

9. Roger VL, Weston SA, Redfield MM, et al. Trends in heart failure incidence and survival in a community-based population. JAMA 2004; 292: 344-50.

10. Heidenreich PA, Albert NM, Allen LA, et al.; American Heart Association Advocacy Coordinating Committee, Council on Arteriosclerosis, Thrombosis and Vascular Biology, Council on Cardiovascular Radiology and Intervention; Council on Clinical Cardiology; Council on Epidemiology and Prevention; Stroke Council. Forecasting the impact of heart failure in the United States: a policy statement from the American Heart Association. Circ Heart Fail 2013; 6: 606-19.

11. Martindale JL, Wakai A, Collins SP, et al. Diagnosing acute heart failure in the Emergency Department: a systematic review and meta-analysis. Acad Emerg Med 2016; 23: 223-42.

12. Platz E, Merz AA, Jhund PS, Vazir A, Campbell R, McMurray JJ. Dynamic changes and prognostic value of pulmonary congestion by lung ultrasound in acute and chronic heart failure: a systematic review. Eur J Heart Fail 2017; 19: 1154-63.

13. Miglioranza MH, Picano E, Badano LP, et al. Pulmonary congestion evaluated by lung ultrasound predicts decompensation in heart failure outpatients. Int J Cardiol 2017; 240: 271-8.

14. Volpicelli G, Elbarbary M, Blaivas M, et al.; International Liaison Committee on Lung Ultrasound for International Consensus Conference on Lung Ultrasound (ILC-LUS) for International Consensus Conference on Lung Ultrasound (ICC-LUS). International evidence-based recommendations for point-of-care lung ultrasound. Intensive Care Med 2012; 38: 577-91.

15. Spinelli A, Vinci B, Tirella A, et al. Realization of a poroelastic ultrasound replica of pulmonary tissue. Biomatter 2012; 2: 37-42.

16. Wabel P, Chamney P, Moissl U, Jirka T. Importance of whole-body bioimpedance spectroscopy for the management of fluid balance. Blood Purif 2009; 27: 75-80.

17. Raimann JG, Zhu F, Wang J, et al. Comparison of fluid volume estimates in chronic hemodialysis patients by bioimpedance, direct isotopic, and dilution methods. Kidney Int 2014; 85: 898-908.

18. Onofriescu M, Hogas S, Voroneanu L, et al. Bioimpedance-guided fluid management in maintenance hemodialysis: a pilot randomized controlled trial. Am J Kidney Dis 2014; 64: 111-8.

19. Covic A, Ciumanghel Al, Siriopol D, et al. Value of bioimpedance analysis estimated "dry weight" in maintenance dialysis patients: a systematic review and meta-analysis. Int Urol Nephrol 2017; 49: 2231-45.

20. Rickham PP. Human experimentation. Code of ethics of the World Medical Association. Declaration of Helsinki. Br Med J 1964; 2: 177.

21. Mancia G, Fagard R, Narkiewicz K, et al. 2013 ESH/ESC Guidelines for the management of arterial hypertension: the Task Force for the management of arterial hypertension of the European Society of Hypertension (ESH) and of the European Society of Cardiology (ESC). J Hypertens 2013; 31: 1281-1357. 
22. Levin A, Lamb EJ, White CT, et al. Kidney disease: Improving global outcomes (KDIGO) CKD work group. KDIGO 2012 clinical practice guideline for the evaluation and management of chronic kidney disease. Kidney Int Suppl 2013; 3: 1-150.

23. Schrefer S (ed.). Mosby's Guide to Physical Examination. $3^{\text {rd }}$ ed., 1995; p. 419.

24. Levey AS, Stevens LA, Schmid CH, et al. A new equation to estimate glomerular filtration rate. Ann Intern Med 2009; 150: 604-12.

25. Lang RM, Badano LP, Mor-Avi V, et al. Recommendations for cardiac chamber quantification by echocardiography in adults: an update from the American Society of Echocardiography and the European Association of Cardiovascular Imaging. Eur Heart J Cardiovasc Imaging 2015; 16: 233-70.

26. Jambrik Z, Monti S, Coppola V, et al. Usefulness of ultrasound lung comets as a nonradiologic sign of extravascular lung water. Am J Cardiol 2004; 93: 1265-70.

27. Available at:https://www.fmc-au.com/therapy-systems-andservices/analysis-systems/bcm [Accessed: 22.12.2019].

28. Moissl UM, Wabel P, Chamney PW, et al. Body fluid volume determination via body composition spectroscopy in health and disease. Physiol Meas 2006; 27: 921-33.

29. Grambsch PM, Therneau TM, Fleming TR. Diagnostic plots to reveal functional form for covariates in multiplicative intensity models. Biometrics 1995; 51: 1469-82.

30. Gattis WA, O'Connor CM, Gallup DS, Hasselblad V, Gheorghiade M; IMPACT-HF Investigators and Coordinators. Predischarge initiation of carvedilol in patients hospitalized for decompensated heart failure: results of the Initiation Management Predischarge: Process for Assessment of Carvedilol Therapy in Heart Failure (IMPACT-HF) trial. J Am Coll Cardiol 2004; 43: 1534-41.

31. Gheorghiade M, Konstam MA, Burnett JC Jr, et al. Efficacy of Vasopressin Antagonism in Heart Failure Outcome Study With Tolvaptan I. Short-term clinical effects of tolvaptan, an oral vasopressin antagonist, in patients hospitalized for heart failure: the EVEREST Clinical Status Trials. JAMA 2007; 297: 1332-43.

32. Domenech P, Perez T, Saldarini A, Uad P, Musso CG. Kidney-lung pathophysiological crosstalk: its characteristics and importance. Int Urol Nephrol 2017; 49: 1211-5.

33. Sharkey RA, Mulloy EM, O'Neill SJ. The acute effects of oxygen and carbon dioxide on renal vascular resistance in patients with an acute exacerbation of COPD. Chest 1999; 115: 1588-92.

34. Gustafsson M, Alehagen U, Johansson P. Imaging congestion with a pocket ultrasound device: prognostic im plications in patients with chronic heart failure. J Card Fail 2015; 21: 548-54.

35. Platz E, Lewis EF, Uno $\mathrm{H}$, et al. Detection and prognostic value of pulmonary congestion by lung ultrasound in ambulatory heart failure patients. Eur Heart J 2016; 37 : 1244-51.

36. Dwyer KH, Merz AA, Lewis EF, et al. Pulmonary congestion by lung ultrasound in ambulatory patients with heart failure with reduced or preserved ejection fraction and hypertension. J Card Fail 2018; 24: 219-26.

37. Park CS, Lee SE, Cho HJ, et al. Body fluid status assessment by bio-impedance analysis in patients presenting to the emergency department with dyspnea. Korean J Intern Med 2018; 33: 911-21.

38. Parrinello G, Paterna S, Di Pasquale P, et al. The usefulness of bioelectrical impedance analysis in differentiating dyspnea due to decompensated heart failure. J Card Fail 2008; 14: 676-86.
39. Siriopol D, Hogas S, Voroneanu L, et al. Predicting mortality in haemodialysis patients: a comparison between lung ultrasonography, bioimpedance data and echocardiography parameters. Nephrol Dial Transplant 2013; 28: 2851-9. 